\title{
NUEVA VARIEDAD DE TRIGO (Triticum aestivum L.) PANADERO: 'MS INTA BONAERENSE 514'
}

\section{NEW CULTIVAR OF BREAD WHEAT (Triticum aestivum L.): ‘MS INTA BONAERENSE 514'}

\author{
Francisco Javier Di Pane ${ }^{1 *}$, Elena Rosa Molfese ${ }^{1}$, María Laura Seghezzo ${ }^{1}$, Gilberto Kraan ${ }^{1}$ \\ ${ }^{1}$ Chacra Experimental Integrada Barrow, INTA-MAIBA, Ruta 3- Km 487,5, Tres Arroyos - CP: 7500- \\ CC: 50 - República Argentina. \\ * Autor para correspondencia E-mail: dipane.francisco@inta.gob.ar
}

\section{RESUMEN}

La nueva variedad de trigo (Triticum aestivum L.) MS INTA BONAERENSE 514 (MS INTA BON 514) es una variedad de trigo rojo panadero de crecimiento primaveral, designada en la estación experimental como W08033, desarrollada y liberada por la Estación Experimental Barrow en 2014, Argentina. 'MS INTA BON 514' es una línea derivada de una F2 desde la cruza Baguette 10/W99008 realizada por el Programa de Mejoramiento de Trigo para Pan en la primavera de 2002. Baguette 10 es un trigo panadero rojo primaveral liberado por Nidera en 1999 , con alto potencial de rendimiento y susceptible a roya de la hoja (Puccinia triticina Eriks.), y la línea élite W99008 que es un trigo panadero rojo de primavera con alta calidad. 'MS INTA BON 514' fue liberada por su excelente potencial de rendimiento a través de su evaluación en un amplio rango de ambientes trigueros en Argentina. Se caracteriza por ser resistente a vuelco, tener maduración temprana, ser resistente a la roya del tallo (Puccinia graminis Pers.) e intermedia resistencia a roya de la hoja (Puccinia triticina Eriks), y poseer una excelente calidad industrial. El nuevo cultivar se adapta a áreas húmedas y sub húmedas de la región pampeana y las tierras más cálidas (noroeste de Argentina). Fue categorizada por el Comité de Cereales de Invierno de la Comisión Nacional de Semillas (CONASE) en 2015, como una variedad del Grupo 1 (trigo corrector). El cultivar se comercializará por la empresa Macro Seed en un convenio con el Instituto Nacional de Tecnología Agropecuaria (INTA).

Palabras clave: cultivar, calidad, trigo panadero, Argentina.

\begin{abstract}
The new variety MS INTA BON 514, which is a hard red spring wheat (Triticum aestivum $\mathrm{L}$.) cultivar with the experimental designation W08033, was developed and released by the Experimental Barrow Station (Argentina) in 2014. 'MS INTA BON 514' is an F2-derived line from the cross Baguette 10/W99008 made in the Bread Wheat Breeding Program in the spring of 2002. Baguette 10 is a hard red spring wheat cultivar released by Nidera in 1999, which has a high yield potential but it is susceptible to leaf rust (Puccinia triticina Eriks.); W99008 is a hard red spring wheat elite line with high quality. 'MS INTA BON 514' was released for its excellent grain potential across a wide range of wheat environments in Argentina. It is characterized by good resistance to lodging, early maturing, good resistance to stem rust (Puccinia graminis Pers.), intermediate resistance to leaf rust, and excellent industrial quality. It adapts well to humid and sub humid areas, such as the
\end{abstract}

Recibido: 01 julio $2016 . \quad$ Aceptado: 28 octubre 2016. 
Pampa region, and warmer zones (northwestern Argentina). In 2015, it was categorized as a variety of the Group 1 (wheat corrector) by the Winter Cereals Committee of the National Seeds Commission (CONASE). 'MS INTA BON 514' will be commercialized by Macro Seed through an agreement with the National Institute of Agricultural Technology (INTA).

Key words: cultivar, quality, bread wheat, Argentina.

\section{ORIGEN, PROCESO DE SELECCIÓN Y ZONA DE DIFUSIÓN}

El cultivo de trigo para pan en Argentina se realiza en una vasta zona que se divide en nueve subregiones trigueras, desde las regiones Noroeste y Noreste argentinos (NOA y NEA) hasta el Sur de la zona pampeana (IV y V Sur). Estas subregiones tienen características agroecológicas diferenciales (Zarilli, 2010). La Chacra Experimental Integrada Barrow (CEI Barrow) (38 20’ S; $60^{\circ} 13^{\prime} \mathrm{W}$ ), que funciona bajo un Convenio entre INTA y MAIBA (Instituto Nacional de Tecnología Agropecuaria y Ministerio de Agroindustria de la provincia de Buenos Aires), está ubicada en Tres Arroyos, subregión IV, Buenos Aires, Argentina.

La nueva variedad MS INTA BON 514 (Res. № 15244) (INASE, 2015) fue obtenida en el Programa de Mejoramiento de Trigo para pan que se lleva adelante en éste lugar. Es una línea derivada del cruzamiento entre el cultivar Baguette 10 (Arche/Genial) (INASE, 1999) de alto rendimiento pero de reducida calidad industrial y susceptible a enfermedades foliares, y una línea élite del programa de trigo pan de la CEI Barrow, W99008 (LAP1438/MILAN//BPONCHO (INASE 1986)) de alta calidad comercial e industrial. El número del cruzamiento y el historial de selección es: Bw 5252-0-5-0-3-2-0W/08. La selección se realizó en forma genealógica y masal (Atlin et al., 2001) en forma intercalada, desde F2 a F6 (Tabla 1).

Fue probada durante tres años en ensayos propios de producción de grano. Se seleccionaron
5 líneas en F6 de las cuales una de ellas, designada como W08033, se destacó por rendimiento, aptitud en calidad panadera y sanidad, dando origen a 'MS INTA BON 514'.

Previo a su registro, la línea fue analizada en ensayos comparativos de rendimiento (ECR), con diseño en bloques completos aleatorizados utilizando como testigos los cultivares Buck Meteoro (INASE, 2008), SY 100 (INASE, 2010) y Biointa 3005 (INASE, 2009), correspondiente a los Grupos 1, 2 y 3 de Calidad (Miranda et al., 2001), respectivamente, los cuales fueron utilizados para categorizar la nueva variedad.

En Argentina para la inscripción de nuevos materiales, la CONASE exige que éstos sean incluidos en ECR junto a testigos que correspondan a cada uno de los Grupos de Calidad. Fue incluida en Ensayos Regionales en las Estaciones Experimentales Agropecuarias (EEA) del INTA de Marcos Juárez, Paraná, Pergamino, Bordenave, Balcarce, la Chacra de Miramar (perteneciente al Ministerio de Asuntos Agrarios de la provincia de Buenos Aires) y el campo anexo en Corral de Bustos (Córdoba). De acuerdo al historial de selección y las pruebas efectuadas se considera apto para sembrar en la región pampeana húmeda, subhúmeda-semiárida, Noroeste y Noreste de Argentina. Los datos se analizaron mediante ANOVA y con prueba de Fisher para diferencias entre medias.

La evaluación de la calidad comercial e industrial se realizó en el Laboratorio de Calidad Industrial de Granos de la CEI Barrow.

Los parámetros y metodologías utilizadas,

Tabla 1. Caracterización molecular de la variedad MS INTA BONAERENSE 514. Se destaca su perfil de gluteninas, crecimiento primaveral e insensibilidad al fotoperíodo.

Table 1. Molecular characterization of the MS INTA BON 514 cultivar (See the gluten-profile, spring growth and photoperiod-sensitivity).

\begin{tabular}{|c|c|c|c|c|c|c|c|c|c|c|c|}
\hline & Glu-A1 & Glu-B1 & Glu-D1 & Vrn-A1 & Vrn-B1 & Vrn-D1 & $\begin{array}{l}\text { Vernali- } \\
\text { zación }\end{array}$ & Ppd-B1 & Ppd-D1 & Fotoperíodo & Lr34 \\
\hline $\begin{array}{l}\text { MS } \\
\text { INTA } \\
\text { Bon } 514\end{array}$ & nulo & $7 \mathrm{oe}+8$ & $5+10$ & S & W & W & Primaveral & $\mathrm{s}$ & I & Insensible & - \\
\hline
\end{tabular}

Glu-A1; Glu-B1 y Glu-D1: Gluteninas de Alto Peso Molecular (GAPM) del genoma A, B y D respectivamente.

Vrn-A1; Vrn-B1 y Vrn-D1: Genes de vernalización de los genomas A, B y D, respectivamente; S: spring (primaveral); $\mathrm{W}$ : winter (invernal);

Ppd-B1 y Ppd-D1: Genes de sensibilidad al fotoperiodo de los genomas B y D, respectivamente; s: sensible; i: insensible. Lr34: Gen menor de resistencia a roya de la hoja. 
según Normas aprobadas, se describen a continuación:

Peso hectolítrico $\left(\mathrm{kg} \mathrm{hL}^{-1}\right)$ : peso específico de una partida de trigo. Resolución SAGPyA № 557/97.

Proteína (\%): contenido de nitrógeno multiplicado por 5,7, expresado tomando como base 13,5\% de humedad. Método NIRT.

Cenizas (\%): contenido de sales minerales, expresado como sustancia seca (IRAM 15.851:2009

Dureza: de acuerdo al método de índice de partícula (PSI)

Molienda: se realizó en un molino experimental Bühler que simula el proceso industrial, obteniéndose el valor de rendimiento harinero en $\%$.

Relac. Mol.: relación rendimiento/ceniza harina $(\%)$.

Humedad (\%): se efectuó secando la muestra en una estufa de circulación forzada de aire, durante una hora (IRAM 15850-I: 2009).

Falling Number o Número de caída (segundos): indica la actividad de la enzima alfa-amilasa en la harina (IRAM 15.862:2003).

Color: color harina (valor b) Minolta Chroma Meter CR-310.

Gluten húmedo (\%): contenido de gluten de la harina (IRAM 15.864-2*:2013).

Alveograma (IRAM 15.857:2012): W: energía de la masa $\left(\mathrm{J} \times 10^{-04}\right)$ y $\mathrm{P} / \mathrm{L}$ : relación tenacidad/ extensibilidad; P: tenacidad de la masa, indica la resistencia a ser estirada o deformada; L: extensibilidad de la masa, indica capacidad para permitir estiramiento.

Farinograma (IRAM 15.855:2000): AA: \% Absorción de agua (base 14,0\% humedad); TD: Tiempo de desarrollo (min); EST: Estabilidad (min); Indice de Calidad Farinográfico (FQN).

Panificación Los panes se elaboraron según la Norma IRAM 15.858- 1 y 2 (1996), que describe un ensayo de panificación experimental para ser usado en programas de mejoramiento de trigo. Se determina Porcentaje de Absorción de Agua (\%AA); Tiempo de Fermentación (TF) min; Volumen de Pan (Vol) mL, Volumen Específico (Ve) y Valor Panadero (VP)

Con los datos obtenidos se calcula un Índice de Calidad (IC), donde algunos de los parámetros (peso hectolítrico, porcentaje de proteína del grano, relación molinera, porcentaje de gluten húmedo, $\mathrm{W}$ alveográfico, estabilidad farinográfica y volumen de pan) se multiplican por un factor de ponderación según su importancia y se asocia por medio de una fórmula (Miranda et al., 2001).

En Argentina las variedades de trigo se clasifican en tres Grupos de Calidad. Para ello, la CONASE (Comité de Cereales de Invierno), categoriza anualmente las variedades de trigo pan presentadas a inscripción según su calidad en al- guno de estos tres grupos:

GRUPO 1: Trigos correctores para panificación industrial;

GRUPO 2: Trigos para panificación tradicional (más de 8 horas de fermentación);

GRUPO 3: Trigos para panificación directa (menos de 8 horas de fermentación)

La línea y variedades testigos se compararon utilizando esta categorización.

\section{RESULTADOS}

\section{Ciclo del cultivar y rendimiento}

Es un cultivar de ciclo intermedio, porte juvenil semi-erecto, con buen crecimiento inicial y buena sanidad durante todo el ciclo del cultivo. El ciclo de emergencia a floración plena en siembras para grano es de 109 días, y de 144 días a madurez de cosecha, dependiendo de las condiciones climáticas del año. Es insensible al fotoperíodo y sin moderados requerimientos de frío (Tabla 1). La altura de planta a la madurez es de $85 \mathrm{~cm}$, dependiendo principalmente de la disponibilidad de agua durante el ciclo vegetativo. Variedad similar al cultivar SY 100 en altura, pero de ciclo emergencia-floración un día más corto, y a Buck Meteoro siendo $3 \mathrm{~cm}$ menos en altura, y de igual ciclo emergencia-floración. Tamaño y color de hoja verde oscuro, similar a Biointa Bonaerense 2001 (INASE, 2004); de pilosidad en hojas como Biointa 2002 (INASE, 2006) y forma y tamaño de cariopse similar a Baguette 10.

La fecha de siembra adecuada para la Subregión triguera IV y V Sur es la primera quincena de junio. En las Subregiones trigueras II, III y V Norte (Zarilli, 2010) se recomienda adelantar la siembra 15 días con respecto a las primeras mencionadas. La densidad de siembra se debe ajustar para obtener 300 plantas $\mathrm{m}^{-2}$ a la emergencia. En la caracterización molecular, posee en la subunidad B1 de gluteninas de alto peso molecular los alelos $7 \mathrm{e}+8$ (Tabla 2) y las combinaciones de $5+10$ en la subunidad D1, que le confiere excelente cualidades molineras y panaderas (Vanzetti et al., 2013). Es resistente a roya del tallo (Puccinia graminis) y moderadamente resistente a roya de la hoja (P. triticina). Tiene buen comportamiento a manchas foliares. No contiene el gen Lr 34 de "lento enroyamiento" (slow rusting) (Campos, 2008) (Tabla 1). La producción de granos difirió significativamente de otros cultivares como Buck Meteoro y SY 100 en el año 2012, superando al promedio de los testigos en 3 años de evaluación en $270 \mathrm{~kg} \mathrm{ha}^{-1}$ (Tabla 3). MS INTA BON 514 tiene color verde grisáceo en estado vegetativo, tallo amarillo, forma del nudo cuadrado, sin serosidad, entrenudos huecos, espiga inclinada, blanca, semilarga. Con un grano elíptico, de color rojo, 
Tabla 2. Promedio de tres años de análisis $(2012,2013$ y 2014) de los parámetros de calidad comercial e industrial de la nueva variedad MS INTA BON 514 y de tres testigos.

Table 2. Average values for both commercial and industrial quality parameters recorded in MS INTA BON 514 and three control treatments (years 2012, 2013 and 2014).

\begin{tabular}{|c|c|c|c|c|c|c|c|c|c|c|}
\hline \multirow[b]{2}{*}{ Categoría } & \multirow[b]{2}{*}{ Entrada } & \multicolumn{4}{|c|}{ Grano } & \multicolumn{2}{|c|}{ Molienda } & \multicolumn{2}{|c|}{ Harina } & \multirow[b]{2}{*}{ FN } \\
\hline & & Rend. & $\mathrm{PH}$ & PMG & PROT & $\begin{array}{l}\text { Rend. } \\
\text { Har. }\end{array}$ & $\begin{array}{l}\text { Ceniza } \\
\text { harina }\end{array}$ & $\begin{array}{l}\text { Relación } \\
\text { Molinera }\end{array}$ & $\begin{array}{l}\text { Gluten } \\
\text { Húmedo }\end{array}$ & \\
\hline & & $\mathrm{kg} \mathrm{ha}^{-1}$ & $\mathrm{~kg} \mathrm{hL}^{-1}$ & $\mathrm{~g}$ & $\%$ & $\%$ & $\%$ & $\%$ & $\%$ & seg \\
\hline INÉDITO & MS INTA BON 514 & 5712 & 80,4 & 37,7 & 12,6 & 68,3 & 0,442 & 156,6 & 31,7 & 404 \\
\hline TESTIGO & BUCK METEORO & 5075 & 82,0 & 34,2 & 12,6 & 68,6 & 0,494 & 139,4 & 32,5 & 410 \\
\hline TESTIGO & SY 100 & 5487 & 82,1 & 36,6 & 12,0 & 69,1 & 0,444 & 156,3 & 31,5 & 378 \\
\hline TESTIGO & BIOINTA 3005 & 5568 & 76,9 & 39,5 & 12,0 & 70,9 & 0,585 & 122,1 & 33,3 & 420 \\
\hline PROMEDIO & TESTIGOS & 5194 & 80,4 & 36,7 & 12,3 & 69,4 & 0,497 & 138,8 & 32,4 & 403 \\
\hline
\end{tabular}

\begin{tabular}{|c|c|c|c|c|c|c|c|c|c|c|c|c|}
\hline \multirow[b]{2}{*}{ Categoría } & \multicolumn{4}{|c|}{ Harina } & \multicolumn{4}{|c|}{ Alveograma } & \multicolumn{2}{|c|}{ Farinograma } & \multicolumn{2}{|l|}{ Pan } \\
\hline & Entrada & $\begin{array}{c}\text { Color, } \\
\text { L }\end{array}$ & $\begin{array}{c}\text { Color, } \\
\text { a }\end{array}$ & $\begin{array}{c}\text { Color, } \\
\text { b }\end{array}$ & W & $P$ & $\mathrm{~L}$ & $\mathrm{P} / \mathrm{L}$ & Estab. & FQN & $\begin{array}{l}\text { Volumen } \\
\text { pan }\end{array}$ & IC \\
\hline INÉDITO & MS IN & 90,53 & $-2,08$ & 8,59 & 367 & 102,0 & 105 & 1,0 & $\begin{array}{l}\text { Min } \\
27,8\end{array}$ & $\begin{array}{c}\mathrm{mm} \\
285,3\end{array}$ & $\begin{array}{c}\mathrm{mL} \\
620\end{array}$ & 65,3 \\
\hline TESTIGO & BUCK METEORO & 90,53 & $-2,30$ & 8,98 & 454 & 112,7 & 116,0 & 1,0 & 25,5 & 360,3 & 712 & 72,3 \\
\hline TESTIGO & SY 100 & 90,63 & $-2,11$ & 8,90 & 314 & 90,7 & 103,3 & 0,88 & 19,8 & 206,0 & 635 & 60,8 \\
\hline TESTIGO & BIOINTA 3005 & 90,33 & $-1,97$ & 8,62 & 271 & 73,3 & 135,7 & 0,54 & 9,4 & 97,7 & 585 & 37,6 \\
\hline PROMEDIO & TESTIGOS & 90,50 & $-2,13$ & 8,83 & 346 & 92,2 & 118,3 & 0,80 & 18,2 & 221,3 & 643,9 & 59,9 \\
\hline
\end{tabular}

Laboratorio Calidad Industrial de Granos, CEI Barrow, INTA-MAIBA.

${ }^{1}$ PH: Peso hectolítrico (kg hL-1); PMG: Peso de mil granos (g); PROT.: Proteína (base 13,5\% hum.); CEN: Ceniza (sobre sustancia seca); REND. HAR. (\%): Rendimiento harina; RELAC. MOL.: relación rendimiento/ceniza harina (\%); FN: Falling Number harina (seg)

COLOR: Minolta - Método triestímulo Chroma Meter CR -310; GLUTEN \%: HUMEDO (base 14,0\% hum); ALVEOGRAMA: P: Tenacidad; L: extensibilidad; W: Energía; P/L: Relación tenacidad/extensibilidad (Norma IRAM 15857 vigente); FARINOGRAMA: AA: \% EST: Estabilidad (minutos); FQN: Número de calidad (Norma IRAM 15855 vigente); PANIFICACION: VOL: Volumen de pan (mL); 60\% de absorción de agua y 140 min. de tiempo de fermentación; IC: Indice calidad industrial (Comité Cereales Invierno CONASE)

con un escudete de tamaño mediano, cepillo mediano, con surco profundo, peso de 1.000 granos $=28,6 \mathrm{~g}$, y peso hectolítrico $=80,36 \mathrm{~kg} \mathrm{hL}^{-1}$.

\section{Calidad comercial e industrial}

En cuanto a la calidad comercial e industrial, en 3 años de evaluación realizada en el Laboratorio de Calidad Industrial de Granos de la CEI Barrow, la nueva variedad MS INTA BON 514 mostró alto peso hectolítrico, tamaño mediano del grano, excelente relación molinera, buena extracción de harina $(68,3 \%)$, y bajo porcentaje de ceniza $(0,442 \%)$, valores que corresponden a un trigo duro a muy duro.

Esta nueva variedad de trigo posee muy buena fuerza panadera, con un $\mathrm{W}$ alveográfico $(\mathrm{W})$ para el trienio de 367, superior al promedio de los testigos (346). La relación $\mathrm{P} / \mathrm{L}$ de la curva alveográfica $(1,0)$, lo muestra como un trigo perfectamente equilibrado. La absorción de agua del nuevo cultivar fue de 59,6\%, similar al valor promedio de los testigos (59,3\%). El tiempo de desarrollo fue de 18,3 min, similar a Buck Meteoro, variedad de reconocida calidad industrial, pero notablemente más alto que los otros dos testigos. La estabilidad fue de 27,8 min, superior a todos los testigos. El Índice de Calidad Farinográfico (FQN) del nuevo cultivar (285) supera a los testigos SY 100 (206) y BIOINTA 3005 (98), siendo solamente inferior a Buck Meteoro (360). El volumen de pan (Vol) de la nueva variedad fue de $620 \mathrm{~cm}^{3}$, similar a SY 100 , pero levemente inferior al promedio de los testigos $\left(644 \mathrm{~cm}^{3}\right)$. El volumen específico (Ve) fue igual a SY $100(4,5)$ y similar al promedio de los testigos $(4,6)$.

La nueva variedad posee un Índice de Calidad (IC) de 65,3 (Miranda et al., 2001), con características reológicas excelentes, dando un volumen de 
Tabla 3. Valores promedios y significación para producción de granos, peso hectolítrico, peso de mil granos, fecha de espigazón, ciclo vegetativo y altura de la variedad MS INTA BON 514 y testigos, en tres años de evaluación $(2012,2013,2014)$.

Tabla 3. Mean values and significance for grain production, test weight, 1000 grain weight, date of earring, vegetative cycle and plant height of the MS INTA BON 514 variety and three control treatments in three years $(2012,2013,2014)$.

\begin{tabular}{|c|c|c|c|c|c|c|c|c|c|}
\hline $\begin{array}{l}\text { Nuevo cultivar y } \\
\text { testigos }\end{array}$ & $\begin{array}{l}\text { Rendimi- } \\
\text { ento }\end{array}$ & $\begin{array}{l}\text { Dif. } \\
\text { rend }\end{array}$ & $\begin{array}{l}\text { Signifi- } \\
\text { cancia }\end{array}$ & $\begin{array}{l}\text { Dif con } \\
\text { media de } \\
\text { testigos }\end{array}$ & $\begin{array}{c}\text { Peso } \\
\text { hectolí- } \\
\text { trico }\end{array}$ & $\begin{array}{l}\text { Peso de } \\
\text { mil } \\
\text { granos }\end{array}$ & $\begin{array}{c}\text { Fecha } \\
\text { espigazón } \\
(50 \%)\end{array}$ & $\begin{array}{c}\text { Días } \\
\text { emergencia } \\
\text { - espigazón }\end{array}$ & $\begin{array}{l}\text { Altura } \\
\text { de } \\
\text { planta }\end{array}$ \\
\hline & $\mathrm{kg} \mathrm{ha}^{-1}$ & $\mathrm{~kg} \mathrm{ha}^{-1}$ & $\begin{array}{l}\text { test LSD } \\
p<0,05\end{array}$ & $\%$ & $\mathrm{~kg} \mathrm{hL}^{-1}$ & Gramos & - & días & $\mathrm{cm}$ \\
\hline \multicolumn{10}{|l|}{ Año 2011} \\
\hline MS INTA BON 514 & 5440 & & & -170 & 80,60 & 43,1 & 28-oct & 113 & 75 \\
\hline B. METEORO & 4970 & 470 & NS & & 79,80 & 38,6 & 28-oct & 113 & 80 \\
\hline SY 100 & 6011 & -571 & NS & & 80,80 & 39,6 & 28-oct & 113 & 75 \\
\hline BIOINTA 3005 & 5847 & -407 & NS & & 77,00 & 46,5 & 03-nov & 119 & 75 \\
\hline Media Test. & 5610 & & & 100 & 79,20 & 41,5 & & 115 & 77 \\
\hline \multicolumn{10}{|l|}{ Año 2012} \\
\hline MS INTA BON 514 & 6041 & & & 906 & 81,50 & 41,5 & 25 -oct & 108 & 85 \\
\hline B. METEORO & 5051 & 990 & S & & 82,60 & 35,2 & 26-oct & 109 & 85 \\
\hline SY 100 & 4997 & 1044 & $\mathrm{~S}$ & & 82,85 & 43,1 & 26-oct & 109 & 85 \\
\hline BIOINTA 3005 & 5357 & 684 & NS & & 77,00 & 41,3 & 30-oct & 113 & 80 \\
\hline Media Test. & 5135 & & & 100 & 80,82 & 39,8 & & 110 & 83 \\
\hline \multicolumn{10}{|l|}{ Año 2013} \\
\hline MS INTA BON 514 & 5488 & & & 75 & 79,00 & 28,6 & 30-oct & 107 & 95 \\
\hline B. METEORO & 5590 & -102 & NS & & 83,70 & 28,7 & 28-oct & 105 & 100 \\
\hline SY 100 & 5350 & 138 & NS & & 82,60 & 27,0 & 31-oct & 108 & 95 \\
\hline BIOINTA 3005 & 5300 & 188 & NS & & 76,80 & 30,6 & 04-nov & 112 & 90 \\
\hline Media Test. & 5413 & & & 100 & 81,03 & 28,8 & & 108 & 95 \\
\hline \multicolumn{10}{|c|}{ PROMEDIO DE LOS AÑOS 2011-2012-2013 } \\
\hline MS INTA BON 514 & 5656 & & & 270 & 80,36 & 37,7 & 27-oct & 109 & 85 \\
\hline B. METEORO & 5203 & 453 & & & 82,03 & 34,2 & 27-oct & 109 & 88 \\
\hline SY 100 & 5452 & 204 & & & 82,08 & 36,6 & 28-oct & 110 & 85 \\
\hline BIOINTA 3005 & 5501 & 155 & & & 76,93 & 39,5 & 02-nov & 115 & 82 \\
\hline Media Test. & 5386 & 270 & & & 80,35 & 36,7 & 29-Nov & 111 & 85 \\
\hline
\end{tabular}

pan aceptable, que la ubica dentro del Grupo 1 de Calidad, los cuales son considerados como trigos correctores (Tabla 2).

\section{Comportamiento frente a factores adversos}

En los ensayos realizados el nuevo cultivar toleró la sequía invernal, fue resistente a las heladas durante el período vegetativo y susceptible a heladas en floración y llenado de granos. Durante el ciclo vegetativo presentó resistencia a roya de la hoja, pero moderada susceptibilidad en estado reproductivo, y resistencia a roya del tallo (Campos, P. 2008); también fue baja la incidencia de "manchas foliares" (Septoria y Dreschlera). Como característica, no presentó desgrane y tampoco vuelco en llenado de granos ni en pre cosecha. No fue caracterizada su tolerancia a los insectos.

Pureza varietal y comercialización

La pureza varietal se mantiene en el Criadero A5851 que se encuentra en la CEI Barrow que depende del Ministerio de Agricultura de la provincia de Buenos Aires (MAIBA). En forma bianual se realiza selección individual de una planta tipo, se siembran las espigas a surco por parcela para que lo recolectado forme parte de la semilla Fun- 
dación, y desde allí se comienza a multiplicar en parcelas/lotes más grandes. La semilla Fundación se obtiene en lotes de 0,6 $55 \mathrm{~m}$, realizándose periódicamente observaciones durante todo el ciclo, desde floración hasta madurez para eliminar plantas fuera de tipo.

La multiplicación de semilla prebásica y la comercialización en toda la República Argentina la realiza la empresa Macro Seed (Grupo Louis Dreyfus Commodities).

\section{Consideraciones finales:}

Argentina se ha destacado por la calidad comercial e industrial de los trigos, y este nuevo material será un aporte muy interesante para el mercado local e internacional, ya que combina potencial de rendimiento, sanidad y calidad comercial e industrial, además de estar recomendado para una amplia región.

\section{LITERATURA CITADA}

Atlin, G.N., M. Cooper, and A. Bjørnstad. 2001. A comparison of formal and participatory breeding approaches using selection theory. Euphytica 122(3):463-475.

Campos, P.E. 2008. Variabilidad de las poblaciones de royas del trigo en Argentina. Congreso Nacional de Trigo. $7^{\circ}$ Simposio Nacional de Cereales de Siembra Otoño-Invernal. $5^{\circ}$ Encuentro del Mercosur. 2 al 4 de julio 2008. Santa Rosa, INTA La Pampa, Argentina.

INASE. 1986. BUCK PONCHO. $\mathrm{N}^{\circ}$ de registro $\mathrm{N}^{\circ}$ 1649. Variedad de Triticum aestivum (L.) Emend. José Buck S.A., Argentina.

INASE. 1999. Baguette 10. $\mathrm{N}^{\circ}$ de registro $\mathrm{N}^{\circ} 6246$. Variedad de Triticum aestivum (L.) Emend. Nidera Semillas S.A., Francia.

INASE. 2004. BIOINTA BONAERENSE 2001. N ${ }^{\circ}$ de registro $\mathrm{N}^{\circ} 7975$. Variedad de Triticum aestivum (L.) Emend. Chacra Exp. Int. Barrow (MAA-INTA), Argentina.
INASE. 2006. BIOINTA 2002. $\mathrm{N}^{\circ}$ de registro $\mathrm{N}^{\circ}$ 9531. Variedad de Triticum aestivum (L.) Emend. INTA, Argentina.

INASE. 2008. BUCK METEORO. $\mathrm{N}^{\circ}$ de registro $N^{\circ}$ 10658. Variedad de Triticum aestivum (L.) Emend. Buck Semillas S.A., Argentina.

INASE. 2009. BIOINTA 3005. $\mathrm{N}^{\circ}$ de registro $\mathrm{N}^{\circ}$ 11563. Variedad de Triticum aestivum (L.) Emend. Inst. Nac. de Tecnología Agropecuaria, Argentina.

INASE. 2010. SY 100. $\mathrm{N}^{\circ}$ de registro $\mathrm{N}^{\circ}$ 11657. Variedad de Triticum aestivum (L.) Emend. Syngenta Seeds Inc., Francia.

INASE. 2015. MS INTA BONAERENSE 514. $\mathrm{N}^{\circ}$ de registro $\mathrm{N}^{\circ} 15244$. Variedad de Triticum aestivum (L.) Emend. Min. Asun. Agr. Pcia. Bs. As./INTA, Argentina.

Miranda, R., y N. Salomón. 2001. Índice de calidad industrial en trigo: una herramienta para determinar la aptitud de los materiales genéticos. Simposio Internacional de Estrategias y Metodologías Utilizadas en el Mejoramiento de Trigo. ISBN: 9974-7586-2-9. M. Kohli, M. Díaz, y M. Castro (eds.). Centro Internacional de Mejoramiento de Maíz y Trigo (CIMMYT), Montevideo, Uruguay.

Vanzetti, L.S., N. Yerkovich, E. Chialvo, L. Lombardo, L. Vaschetto, and M. Helguera. 2013. Genetic structure of Argentinean hexaploid wheat germplasm. Genetics and Molecular Biology 36(3):391-399.

Zarilli, A.G. 2001. Ecología, capitalismo y desarrollo agrario en la región Pampeana (18901950). Un enfoque histórico-ecológico de la cuestión agraria. Disponible en http://www. eumed.net/tesis-doctorales/2010/agz/erosioneolicaylastormentas detierraenlaestepapampeana.htm. (Consulta 18 julio 2015). 